\title{
P02-103
}

\section{PSYCHIATRIC INPATIENT READMISSION IN A GENERAL HOSPITAL: PRELIMINARY REPORT OF A TWO YEARS OBSERVATIONAL STUDY}

D. Guerreiro, R. Navarro, D. Telles, P. Martins, E. Trigo, M. Silva, M. Carvalho, C. Gois

Psychiatry Department, Hospital Santa Maria, Faculdade Medicina Lisboa, Lisbon, Portugal

Introduction: Studying inpatient clinical population's features allows a better understanding of readmissions. Factors associated with higher levels of readmission may give clues concerning better treatment planning and follow-up.

Objectives: To detect psychosocial and clinic factors associated with readmissions in an inpatient general psychiatric population. Method: 107 patients consecutively admitted to an inpatient unit were characterized in terms of psychosocial, clinical, treatment and discharge plan features. Readmission was defined as the number of previous admittances in the last two years.

Results: Patients characteristics: Average age 44 (SD: 16); 58\% female; 10 (SD:5) years of education; average disease duration of 128 (SD:115) months. Main diagnosis: 30,8\% schizophrenia; 14\% bipolar I disorder; 13,1\% major depression; $11,2 \%$ recurrent major depression; $5,6 \%$ cluster B personality; 3,8\% bipolar II disorder and 2,9\% schizoaffective disorder . From more to less frequent diagnosis implicated in readmissions were schizophrenia, schizoaffective disorder, recurrent major depression, bipolar I, bipolar II and major depression episode. Readmissions were not associated with inpatient treatment or disease duration, nor toxics or axis II diagnosis. Nonetheless, beginning a depot antipsychotic medication was related to an increase in previous admissions.

Conclusion: These are preliminary results of a small sample, but they point towards the usefulness of a systematic evaluation of inpatient populations, mainly in what concerns complex clinical situations like major pathology readmissions. 